\title{
An Automated Approach to Differentiate a Normal eye from a Defective Eye
}

\author{
Hillol Das \\ Department of Computer Science and Engineering \\ National Institute of Technology, Agartala, India
}

\author{
Suman Deb \\ Department of Computer Science and Engineering \\ National Institute of Technology, Agartala, India
}

\begin{abstract}
Detection of basic differentiating characteristics of eye diseases from the images of the retina can be a good approach as a low-cost method for broad-based initial screening. For example early diabetic retinopathy detection enables application of laser therapy treatment in order to prevent or delay loss of vision. The paper has referenced Diabetic retinopathy and Retinitis pigmentosa for analysis. Automated approach for detection of microaneurysms in digital color fundus photographs helps ophthalmologist to detect the emergence of its initial symptoms and determine the next action step for the patient. A similar mechanism for automated early disease detection method with respect to the features of the normal eye is proposed. The detection algorithm features identification of black pigments like minute features, microaneurysm and exudate detection and these features extracted can prove to a greater extent as ready instances for defectiveness. A number of images along with the feedback and consultation from the ophthalmologist in this area of medical science has proved to be a great help towards the observation as derived from this mechanism and discussed in the later end of this paper. The proposed mechanism can be extended up to the limit of supervised learning so as to automate the practical feedbacks as obtained from the practitioners.
\end{abstract}

\section{Keywords}

Diabetic Retinopathy, Retinitis Pigmentosa, microaneurysms, opthalmologist.

\section{INTRODUCTION}

Diabetic retinopathy, the most common diabetic eye disease, occurs when blood vessels in the retina change. It is found to be the leading causes of visual impairment in the present day world [5]. Sometimes these vessels swell and leak fluid or even close off completely. In other cases, abnormal new blood vessels grow on the surface of the retina. The longer time a person has diabetes there are greater chances that he/she might suffer from this disease. Diabetic retinopathy usually affects both eyes.

People who have diabetic retinopathy often don't notice changes in their vision in the disease's early stages. But as it progresses, diabetic retinopathy usually causes vision loss that in many cases cannot be reversed.

\author{
Ashim Saha \\ Department of Computer Science and Engineering
National Institute of Technology, Agartala, India \\ National Institute of Technology, Agartala, India
}

Gautam Nath

Department of Computer Science and Engineering

National Institute of Technology, Agartala, India
In the UK diabetic retinopathy is the leading cause of blindness (number 4 of the entire cause of blindness in aged 30-65 years), whereas in the United States there are 5,000 people per year suffer due to diabetic retinopathy [1]. Manifestations of the disease can occur in $80 \%$ of all diabetics who have suffered for over 10 or 15 years and can cause blindness [2].

Retinitis pigmentosa on the other hand is a group of related eye disorders that cause progressive vision loss. These disorders affect the retina, which is the layer of light-sensitive tissue at the back of the eye. In people with retinitis pigmentosa, vision loss occurs as the light-sensing cells of the retina gradually deteriorate.

The first sign of retinitis pigmentosa is usually a loss of night vision, which becomes apparent in childhood. Problems with night vision can make it difficult to navigate in low light. Later, the disease causes blind spots to develop in the side (peripheral) vision. Over time, these blind spots merge to produce tunnel vision. The disease progresses over years or decades to affect central vision and hence early detection can be a preventive step to stop its further growth.

Many methods have been proposed in order to get an accurate blood vessel detection and segmentation in fundus images like multilayered thresholding technique to segment the vessels [3], usage of scale and orientation selective Gabor filters to detect the abnormal blood vessels [4]. The previous automation techniques were limited to a real-time quality measurement of the captured images [6]. Later automation included lesion detection [7, 8], anatomical feature localization [9] and these can be the basis for a supervised disease stratification methodology implementing contentbased image retrieval (CBIR) [10]. In the present work, it tries to devise a detection mechanism which can serve as the quality estimation algorithm to determine the impact the basic identification features based on the thresholds on disease detection data collected. The paper attempts to show the statics of the experimental performance with a handsome set of images [11]. Based on the literature referred and observations from experiments it may be concluded with the findings which stand as symptoms and some future paths for development to serve as advancement in this field of work. 


\section{OVERVIEW OF THE PRESENT WORK}

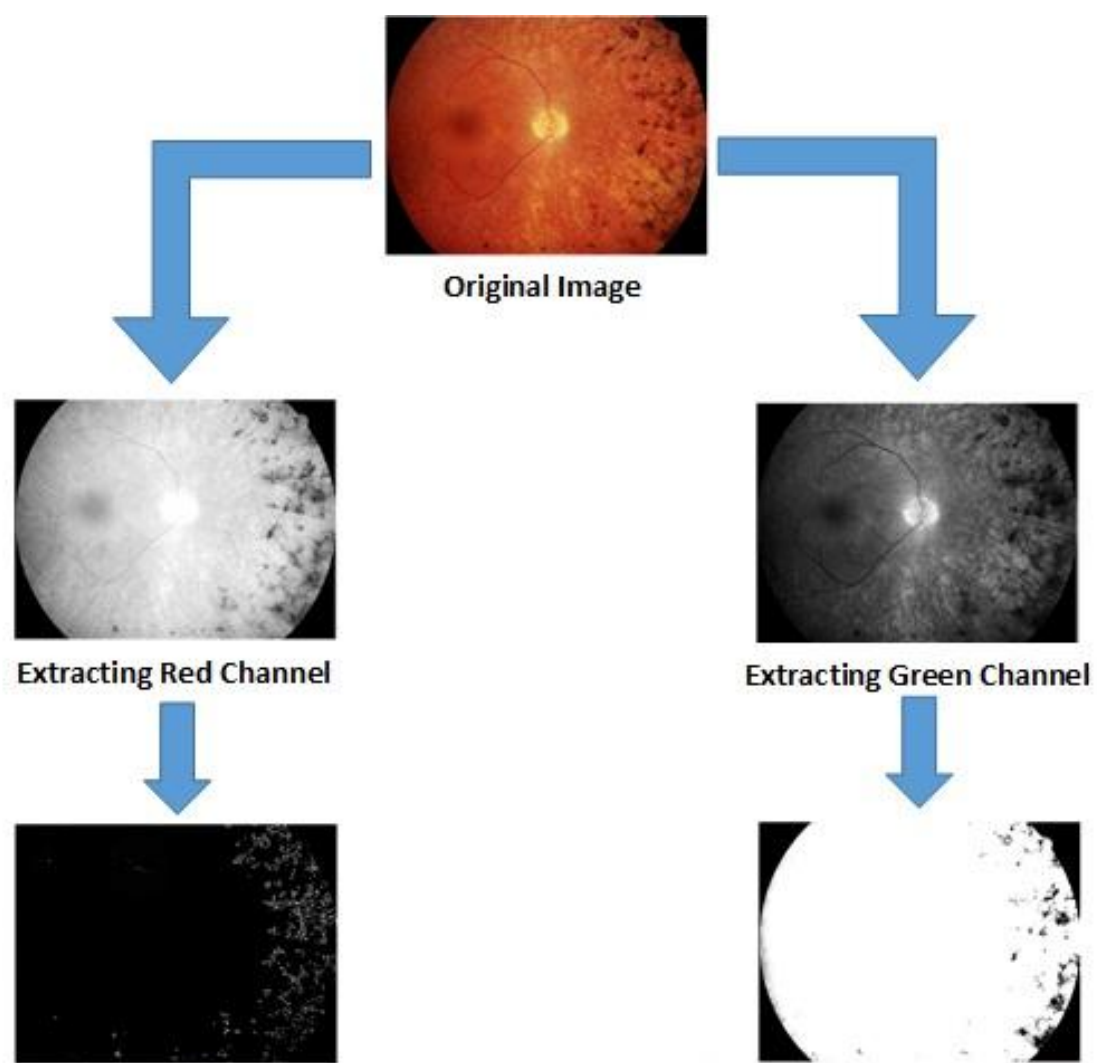

Sobel method edge detection

Region of Interest extracted by simple threshold range (pre-calculated)
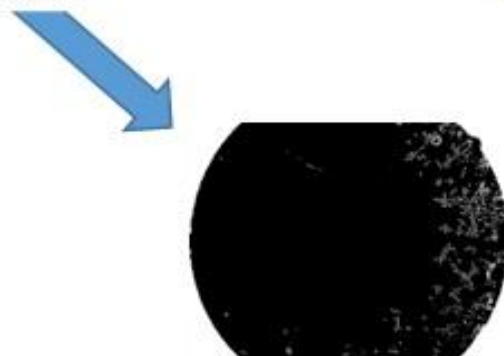

Image fused (only masked area)

Final Image with black pixels highlighted as detection of affected region of retinal fundus image

Here, the image shown is of a patient suffering from Retinit is Pigmentosa

Fig 1: Overview of experimental steps towards the study for automated detection of features in a defective eye. A thorough quality assessment have been performed based on the model overview presented above with a handy set of images to obtain a statistical result set as will be explained in the later part of the experiment. 


\subsection{Image Acquisition}

Source 1 : Such images are obtained from the HighResolution Fundus (HRF) Image Database[11].This database has been established by a collaborative research group to support comparative studies on automatic segmentation algorithms on retinal fundus images. The database is provided by the Pattern Recognition Lab (CS5), the Department of Ophthalmology, Friedrich-Alexander University ErlangenNuremberg (Germany), and the Brno University of Technology, Faculty of Electrical Engineering and Communication, Department of Biomedical Engineering, Brno (Czech Republic).

Source 2: DIARETDB0 - Standard Diabetic Retinopathy Database [13] is the other source of images used for experimental purpose. This is a public database for benchmarking diabetic retinopathy detection from digital images. The main objective of the design has been to unambiguously define a database and a testing protocol which can be used to benchmark diabetic retinopathy detection methods. By using this database and the defined testing protocol, the results between different methods can be compared. The data base is provided by the Machine Vision and Pattern Recognition Research Group, Laboratory of Information Processing, Lappeenranta University of Technology, Finland.

Source 3: A good number of images have been obtained with prior permission from the image database belonging to Indira Gandhi Memorial (IGM) Hospital and Agartala Government Medical College and Hospital located in the State of Tripura (India).

\subsection{Color Plane Extraction}

An RGB image, sometimes referred to as a truecolor image, is stored in MATLAB as an m-by-n-by-3 data array that defines red, green, and blue color components for each individual pixel. RGB images do not use a palette. The color of each pixel is determined by the combination of the red, green, and blue intensities stored in each color plane at the pixel's location.

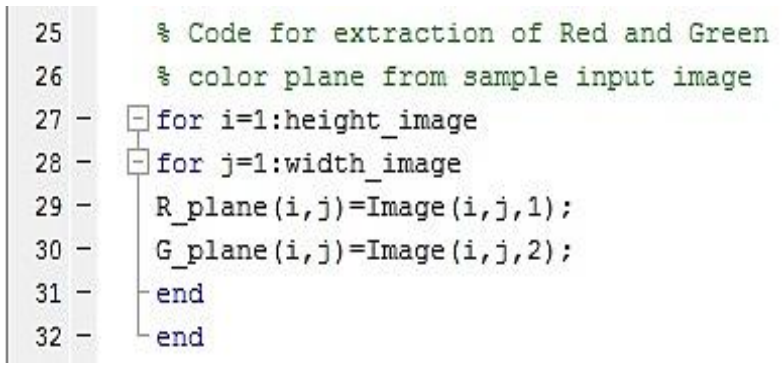

Fig 2: Snapshot of code (MATLAB 7) for extraction of Red \& Green Plane of Input Image.

From survey it has been found that color retinal image has unique characteristic than other image, that is, this image has important in green $(\mathrm{G})$ channel [12] whereas red ( R ) channel servers a better purpose for Sobel edge detection as explained through figure 3 , figure 4 and figure 5.

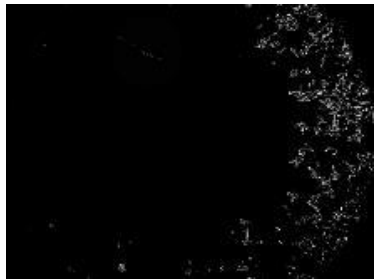

Fig 3: Sobel method applied to Red Plane. This figure serves better for further analysis because of clear detection of defective region.

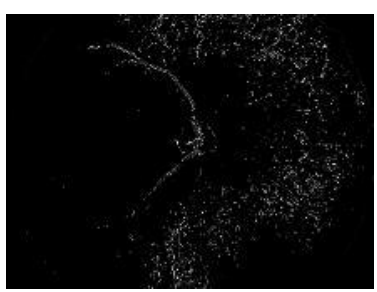

Fig 4: Sobel method applied to Green Plane. This figure exposes the unwanted region (like retina vessels) of retina which is not considered as a part of the current analysis.

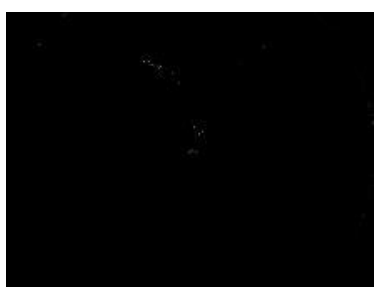

Fig 5: Sobel method applied to Blue Plane. This figure has a very poor visibility for analysis of the defective ranion

\subsection{Sobel method for edge detection}

The Method parameter, selected as Sobel detects block and finds the edges in an red component input image (Figure 2, Figure 3 and Figure 4 explains the reason behind the specific choice of Red Component instead of Green or Blue Component) by approximating the gradient magnitude of the image. The block convolves the input matrix with the Sobel. The block outputs two gradient components of the image, which are the result of this convolution operation. Alternatively, the block can perform a thresholding operation on the gradient magnitudes and output a binary image, which is a matrix of Boolean values. If a pixel value is 1 , it is an edge.

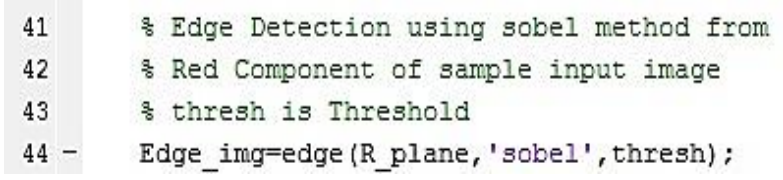

Fig 6: Snapshot of code (MATLAB 7) for detection of edge from the Red Plane image obtained in S tep 2.2.

\subsection{Working with ROI (Region of Interest)}

In this process the region of interest (ROI) is calculated by interpolating the pixel values from the borders of the region. This process can be used to make objects in an image seem to disappear as they are replaced with values that blend in with the background area. With respect to the green component, region of interest are determined using set of threshold values gather on a real time scenario. However this set of threshold values can be subjected to alteration based on a self-learning methodology as a part of future scenario. 


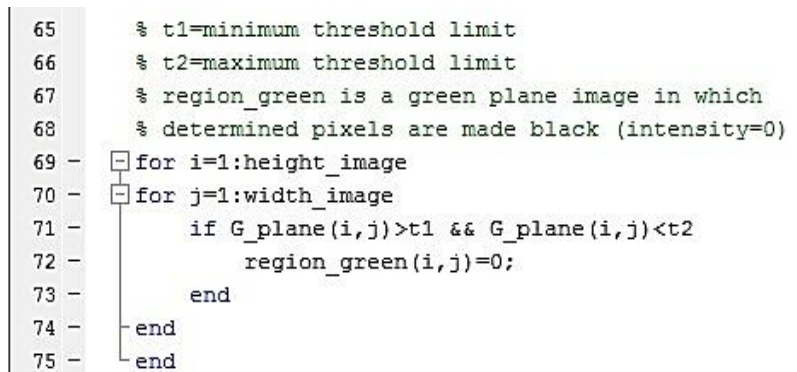

Fig 7: S napshot of code (MATLAB 7) for generating region of interest from the Green Plane image obtained in Step 2.2

\subsection{Fusion of images from 2.3 and 2.4}

For achieving the fusion mechanism the images obtained from step 2.3 and step 2.4 have been plotted to a zero matrix of dimension equal to the input images and the result so obtained displays the affected areas highlighted as white pixels. The Mask as shown in figure 9, is applied on the target image for removal of unwanted region.

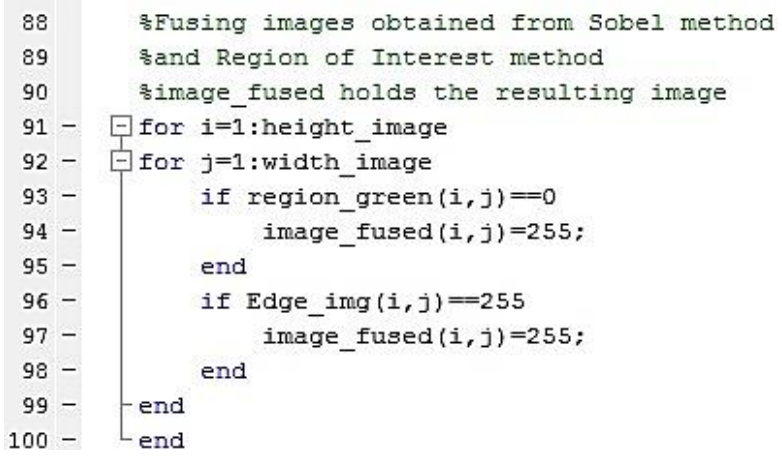

Fig 8: Snapshot of code (MATLAB 7) for implementing fusion.
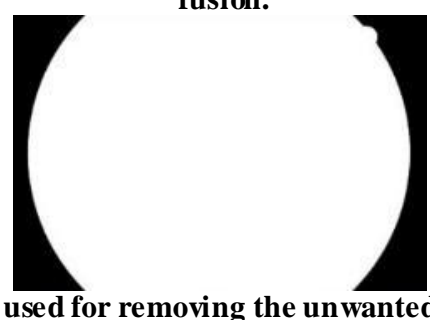

Fig 9: Mask used for removing the unwanted region of the target image.

\subsection{Image Interpolation}

In engineering and science, one often has a number of data points, obtained by sampling or experimentation, which represent the values of a function for a limited number of values of the independent variable. It is often required to interpolate (i.e. estimate) the value of that function for an intermediate value of the independent variable.

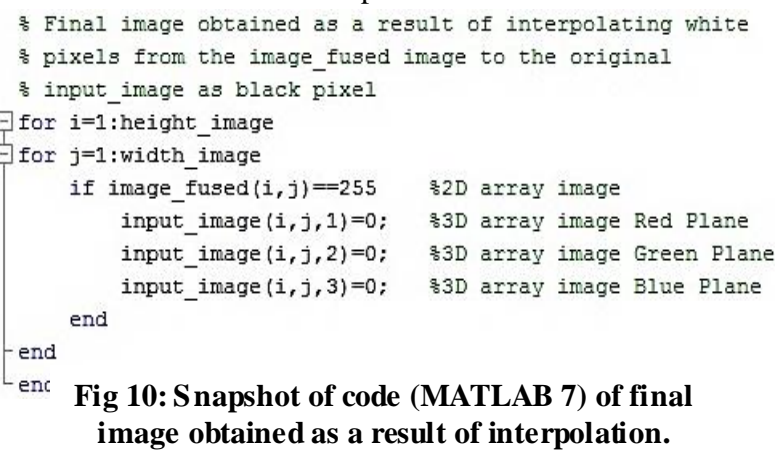

\section{EXPERIMENTAL ANALYSIS}

A detailed experiment has been performed on a total of 85 images collected from open access High-Resolution Fundus (HRF) Image Database [11] and DIARETDB0 - Standard Diabetic Retinopathy Database [13]. The results obtained after application of the proposed work has been tabulated by means of comparison with the ground truth and an overall perfection of $81.17 \%$ has been observed as far as the analysis is concerned.

Table 1. Experimental Results

\begin{tabular}{|c|c|c|c|}
\hline Image Set & $\begin{array}{l}\text { Detected } \\
\text { from the } \\
\text { present } \\
\text { work } \\
\text { (Normal/ } \\
\text { Defective) }\end{array}$ & $\begin{array}{c}\text { Ground } \\
\text { Truth } \\
\text { (Normal/ } \\
\text { Defective) }\end{array}$ & $\begin{array}{l}\text { Matched } \\
\text { (Yes/ No) }\end{array}$ \\
\hline \multicolumn{4}{|c|}{ Images from Source 1} \\
\hline 01_h.jpg & Normal & Normal & Yes \\
\hline 02_h.jpg & Normal & Normal & Yes \\
\hline 03_h.jpg & Normal & Normal & Yes \\
\hline 04_h.jpg & Normal & Normal & Yes \\
\hline 05_h.jpg & Normal & Normal & Yes \\
\hline 06_h.jpg & Normal & Normal & Yes \\
\hline 07_h.jpg & Normal & Normal & Yes \\
\hline 08_h.jpg & Normal & Normal & Yes \\
\hline 09_h.jpg & Normal & Normal & Yes \\
\hline 10_h.jpg & Normal & Normal & Yes \\
\hline 11_h.jpg & Normal & Normal & Yes \\
\hline 12_h.jpg & Normal & Normal & Yes \\
\hline 13_h.jpg & Normal & Normal & Yes \\
\hline 14_h.jpg & Normal & Normal & Yes \\
\hline 15_h.jpg & Normal & Normal & Yes \\
\hline 01_dr.jpg & Normal & Defective & No \\
\hline 02_dr.jpg & Defective & Defective & Yes \\
\hline 03_dr.jpg & Defective & Defective & Yes \\
\hline 04_dr.jpg & Defective & Defective & Yes \\
\hline 05_dr.jpg & Defective & Defective & Yes \\
\hline 06_dr.jpg & Normal & Defective & No \\
\hline 07_dr.jpg & Defective & Defective & Yes \\
\hline 08_dr.jpg & Defective & Defective & Yes \\
\hline 09_dr.jpg & Defective & Defective & Yes \\
\hline 10_dr.jpg & Normal & Defective & No \\
\hline 12_dr.jpg & Defective & Defective & Yes \\
\hline 13_dr.jpg & Defective & Defective & Yes \\
\hline 14_dr.jpg & Normal & Defective & No \\
\hline 15_dr.jpg & Normal & Defective & No \\
\hline \multicolumn{4}{|c|}{ Images from Source 2} \\
\hline 01_Img.jpg & Defective & Defective & Yes \\
\hline 02_Img.jpg & Defective & Defective & Yes \\
\hline 03_Img.jpg & Defective & Defective & Yes \\
\hline 04_Img.jpg & Defective & Defective & Yes \\
\hline 05_Img.jpg & Defective & Defective & Yes \\
\hline 06_Img.jpg & Defective & Defective & Yes \\
\hline 07_Img.jpg & Defective & Defective & Yes \\
\hline 08_Img.jpg & Defective & Defective & Yes \\
\hline 09_Img.jpg & Normal & Defective & No \\
\hline 10_Img.jpg & Defective & Defective & Yes \\
\hline 11_Img.jpg & Defective & Defective & Yes \\
\hline 12_Img.jpg & Defective & Defective & Yes \\
\hline 13_Img.jpg & Defective & Defective & Yes \\
\hline 14_Img.jpg & Defective & Defective & Yes \\
\hline 15_Img.jpg & Defective & Defective & Yes \\
\hline 01_Img.jpg & Defective & Defective & Yes \\
\hline
\end{tabular}




\begin{tabular}{|c|c|c|c|}
\hline 02_Img.jpg & Defective & Defective & Yes \\
\hline 03_Img.jpg & Defective & Defective & $\overline{\text { Yes }}$ \\
\hline 04_Img.jpg & Defective & Defective & $\overline{\text { Yes }}$ \\
\hline 05_Img.jpg & Defective & Defective & Yes \\
\hline 06_Img.jpg & Normal & Defective & No \\
\hline 07_Img.jpg & Normal & Defective & $\overline{\text { No }}$ \\
\hline 08_Img.jpg & Defective & Defective & Yes \\
\hline 09_Img.jpg & Defective & Defective & Yes \\
\hline 10_Img.jpg & Defective & Defective & $\overline{Y e s}$ \\
\hline 11_Img.jpg & Defective & Defective & Yes \\
\hline 12_Img.jpg & Defective & Defective & $\overline{\text { Yes }}$ \\
\hline 13_Img.jpg & Defective & Defective & Yes \\
\hline 14_Img.jpg & Defective & Defective & $\overline{\text { Yes }}$ \\
\hline 15_Img.jpg & Normal & Defective & No \\
\hline \multicolumn{4}{|c|}{ Images from Source 3} \\
\hline Igm_1.jpg & Normal & Defective & No \\
\hline Igm_2.jpg & Normal & Defective & No \\
\hline Igm_3.jpg & Normal & Defective & No \\
\hline Igm_4.jpg & Defective & Defective & $\overline{\text { Yes }}$ \\
\hline Igm_5.jpg & Normal & Defective & No \\
\hline Igm_6.jpg & Defective & Defective & $\overline{\text { Yes }}$ \\
\hline Igm_7.jpg & Defective & Defective & $\overline{\text { Yes }}$ \\
\hline Igm 8.jpg & Defective & Defective & Yes \\
\hline Igm_9.jpg & Defective & Defective & Yes \\
\hline Igm_10.jpg & Defective & Defective & $\overline{\text { Yes }}$ \\
\hline Agmc_1.jpg & Defective & Defective & $\overline{\text { Yes }}$ \\
\hline Agmc_2.jpg & Normal & Defective & No \\
\hline Agmc_3.jpg & Defective & Defective & $\overline{\text { Yes }}$ \\
\hline Agmc_4.jpg & Defective & Defective & Yes \\
\hline Agmc_5.jpg & Normal & Defective & No \\
\hline Agmc_6.jpg & Normal & Defective & No \\
\hline Agmc_7.jpg & Defective & Defective & $\overline{\text { Yes }}$ \\
\hline Agmc_8.jpg & Defective & Defective & $\overline{\text { Yes }}$ \\
\hline Agmc_9.jpg & Defective & Defective & $\overline{\text { Yes }}$ \\
\hline Agmc_10.jpg & Defective & Defective & $\overline{Y e s}$ \\
\hline Agmc_11.jpg & Normal & Normal & Yes \\
\hline Agmc_12.jpg & Normal & Normal & Yes \\
\hline Agmc_13.jpg & Normal & Normal & $\overline{\text { Yes }}$ \\
\hline Agmc_14.jpg & Normal & Normal & $\overline{\text { Yes }}$ \\
\hline Agmc_15.jpg & Normal & Normal & Yes \\
\hline \multicolumn{2}{|c|}{ Total 85 Images } & \multicolumn{2}{|c|}{ Yes $=69, \mathrm{No}=16$} \\
\hline
\end{tabular}

$\%$ Perfection of the present work $=\mathbf{8 1 . 1 7 \%}$

Note: The highlighted rows in the above table indicates the negative cases observed which the algorithm could not detect.

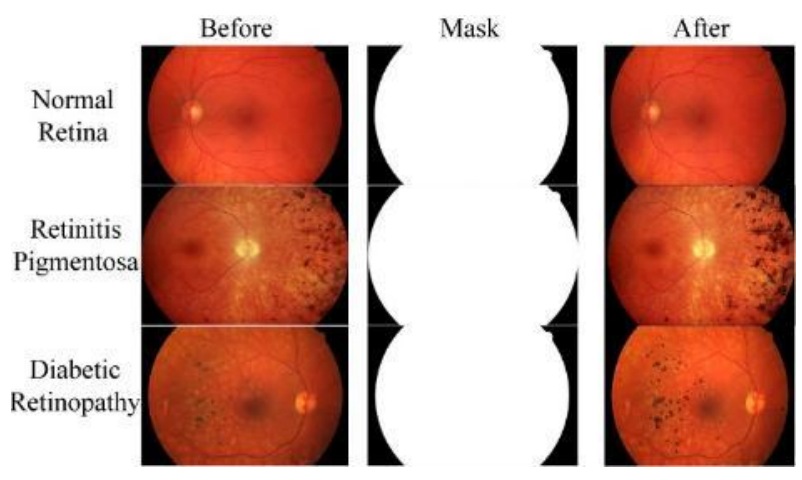

Fig 11: Sample Input \& Output detecting Normal Retina, Retinitis Pigmentosa \& Diabetic Retinopathy Diseases

\section{EXPERIMENTAL CHALLENGES}

-Made use of image database from web sources due to nonavailability of skilled device.

-Variations in image properties obtained from diverse image source configurations posed a great challenge in terms of quality of detection. This may be the reason for which some diseased images produced negative results.

\section{FUTURE SCOPE}

-A self-learning mechanism may be devised to obtain dy namic threshold range.

-Only two types of eye diseases are considered for the present work. Future researchers can extend their scope to several other diseases related to vision.

\section{CONCLUSION}

A widely observed results from the present work helped to get an insight into the perfection of detection. The analysis undertaken could open up doors for the aspiring researchers to come up with improved proposals and explore the detection with an in-depth consideration of all possible minute features.

\section{ACKNOWLEDGEMENTS}

Thanks to Almighty. Also like to extend sincere thanks to the owners of the image database from web sources and to the Hospital Administration of Agartala, India.

\section{REFERENCES}

[1] R. Vidyasari, I. Sovani, and T.L.R. Mengko, H. Zakaria, "Vessel Enhancement Algorithm in Digital Retinal Fundus Microaneurysms Filter for Nonproliferative Diabetic Retinopathy Classification”, 2011 International Conference on Instrumentation, Communication, Information Technology and Biomedical Engineering 89 November 2011, Bandung, Indonesia.

[2] Langston, DB. (1988). "Manual of Ocular Diagnosis and Therapy”, Little Brown Company, Boston.

[3] M.U. Akram, I. Jamal, A. Tariq, J. Imtiaz, “Automated segmentation of blood vessels for detection of proliferative diabetic retinopathy", Biomedical and Health Informatics (BHI), IEEE-EMBS International Conference on, vol., no., pp.232-235, 5-7, 2012.

[4] D. Vallabha, R. Dorairaj, K. Namuduri, H. Thompson, "Automated detection and classification of vascular abnormalities in diabetic retinopathy", Signals, Systems and Computers, Conference Record of the Thirty-Eighth Asilomar Conference on, vol.2, pp.1625-1629 Vol.2, Nov.2004.

[5] M. A. Bamashmus, B. Matlhaga and G. N. Dutton, "Causes of blindness and visual impairment in the west of scotland", Eye, vol. 18, pp. 257-261, 2004.

[6] Giancardo, L., Abramoff, M.D., Chaum, E., Karnowski, T.P., Meriaudeau, F., and Tobin, K. W., (2008), "Elliptical local vessel density: a fast and robust quality metric for fundus images", 30th Annual International Conf. of the IEEE EMBS Van couver, Can ada, 2008.

[7] Giancardo, L., et. al, (2010), "Microaneurysms detection with the Radon Cliff operator in retinal fundus images", Proc. SPIE Medical Imaging, San Diego CA, 7623:7623. 
[8] Giancardo, L., et. al, (2011), "Automatic retina exudates segmentation without a manually labeled training set", Proc. IEEE International Symposium on Biomedical Imaging.

[9] Tobin KW, Chaum E, Govindasamy VP, Karnowski TP. (2007), "Detection of anatomic structures in human retinal imagery", IEEE Trans Med Imaging 26:1729-39.

[10] Chaum, E., Karnowski, T.P., Govindasamy, V.P., Abdelrahamen M, Tobin K.W., (2008), "Automated diagnosis of retinopathy by content based image retrieval", Retina 28:1463-1477.
[11] http://www5.cs.fau.de/research/data/fundus-images/

[12] Agung W. Setiawan, Tati R. Mengko, Oerip S. Santoso and Andriyan B. Suksmono, "Color Retinal Image Enhancement using CLAHE”, 2013 International Conference on ICT for Smart Society (ICISS).

[13] http://www2.it.lut.fi/project/imageret/diaretdb0/index.ht m 